\title{
Diffusion coefficient, correlation function and power spectral density of velocity fluctuations in monolayer graphene
}

\author{
R. Rengel ${ }^{1, \text { a) }}$ and M. J. Martín ${ }^{1}$ \\ Department of Applied Physics, University of Salamanca, Salamanca, 37008, \\ Spain
}

(Dated: 2 September 2013)

In this paper, the diffusivity in suspended monolayer graphene at low and high electric fields is investigated. The knowledge of this quantity and its dependence on the electric field is of primary importance not only for the investigation of the electronic transport properties of this material, but also for the development of accurate drift-diffusion models. The results have been obtained by means of an ensemble Monte Carlo simulation. For the calculation of the diffusion coefficient, two different methods are considered, one based on the second central moment and the other one based on the Fourier analysis of velocity fluctuations, which are directly related to the noise behaviour at high frequencies. The diffusion coefficient is analyzed considering both parallel and transversal directions with regard to the applied field. Taking into account the importance of degeneracy in this material, the calculations are properly performed by considering an excess electron population obeying a linearized Boltzmann transport equation, which allows studying in an adequate fashion the diffusivity phenomena. The results show the importance of degeneracy effects at very low fields, in which transport is mainly dominated by acoustic phonon scattering. Values of the diffusion coefficient larger than $40.000 \mathrm{~cm}^{2} / \mathrm{Vs}$ are obtained for a carrier concentration equal to $10^{12} \mathrm{~cm}^{-2}$. The correlation function of instantaneous velocity fluctuation is explained in terms of the wavevector distribution, and their power spectral density is evaluated in the $\mathrm{THz}$ range, showing an important dependence on the applied field and being strongly related to microscopic transport processes.

PACS numbers: 72.80.Vp, 72.10.Di, 05.10.Ln

\section{INTRODUCTION}

Due to its extraordinary electronic properties, in the last few years graphene has probably become the most promising material to be considered in the post-Silicon scenario. ${ }^{1-4}$ Measured intrinsic cut-off frequencies of 300 $\mathrm{GHz}$ have been reported in graphene transistors, and estimations from static measurements and simulations indicate that values well exceding the $\mathrm{THz}$ could be reached, thus outperforming traditional semiconductors for analog applications. $^{5-7}$ Graphene nanoribbon transistors have been proposed also as the building blocks for low-noise amplifiers. ${ }^{8} \mathrm{THz}$ emission and detection is also being investigated in order to design new devices who may function as detectors, mixers, phase shifters and frequency multipliers, thanks to the properties of carrier relaxation dynamics and surface plasmons in single layer and multilayer graphene. ${ }^{9-12}$

Therefore, the investigation of the transport properties in graphene up to the $\mathrm{THz}$ range is of primary importance. Moreover, the study of the high-frequency noise properties of this exciting material is crucial for the development of analog applications. Several works have been presented discussing the influence of $1 / f$ noise in monolayer, bilayer graphene and graphene FETs. ${ }^{13-17}$ Recently, high-frequency noise has been studied in graphene on sapphire, showing, in that case, the importance of shot

a) Electronic mail: raulr@usal.es noise associated with hole jumps across the potential barriers located in the graphene layer. ${ }^{18}$

An in-depth study of diffusivity in this material is also a must; in the case of bilayer graphene (which bandstructure notably differs from that of monolayer graphene ${ }^{3}$ ), the electric field dependence of the diffusion coefficient has been studied analytically by using the relaxation time approximation (assuming a constant $\tau$ ) in order to solve the kinetic Boltzmann transport equation. ${ }^{19}$ Other studies have been devoted to monolayer graphene, investigating by pump-probe spectroscopy the expansion of a Gaussian spatial profile of carriers; in this way, diffusion coefficients equal to 11000 and $5500 \mathrm{~cm}^{2} / \mathrm{s}$ in epitaxial graphene and reduced graphene-oxide samples have been observed. ${ }^{20,21}$ The influence of disorder on the diffusion coefficient has been analyzed theoretically by means of a random gap model, both in bilayer and monolayer graphene. ${ }^{22}$ However, in general the diffusion coefficient in monolayer graphene has been mainly described by elementary equations derived considering the classical motion of electrons in a diffusive random walk in equilibrium conditions, ${ }^{3,23}$ not taking into account the influence of the applied electric field. The knowledge of the electric field dependence of the diffusion coefficient in graphene is therefore critical for the development of accurate driftdiffusion models for device simulation. ${ }^{24}$

In the case of a uniform and infinite ideal graphene sheet, several authors have shown the accuracy of the semiclassical model (slightly away form the Dirac point) for the study of this material. ${ }^{25-27}$ Consequently, the Boltzmann transport equation can be steadily solved by 
considering the stochastic movement of an ensemble of carriers under the action of an applied electric field, and different Monte Carlo models have been develped for the study of graphene at the material and device level. ${ }^{27-38}$ While the Monte Carlo technique has already provided accurate and reliable results for the study of the electric field dependence of diffusion coefficients and correlation functions and power spectral density of instantaneous velocity fluctuations in conventional semiconductors, ${ }^{39-42}$ to the authors' knowledge this modelling technique has not been yet employed for a detailed investigation of the diffusivity or high frequency instantaneous velocity fluctuations in graphene.

In this paper we present a Monte Carlo study of diffusivity and carrier velocity fluctuations in monolayer graphene. Particular attention is paid to the correlation functions of velocity fluctuations and their power spectral density up to the $\mathrm{THz}$ range, together with a detailed description of the diffusion coefficient (in the parallel and perpendicular directions with regard to the applied electric field) at low and high electric fields.

\section{SIMULATION PROCEDURE}

\section{A. Monte Carlo model}

The conical shape of the graphene bandstructure close to the Dirac points (the band minima) is taken into account in our model, thus providing a lineal dispersion relationship. ${ }^{43}$ The scattering rates included in the simulator have been derived by other authors taking into account the results obtained by means of $a b$ initio calculations. ${ }^{44,45}$ The parameters (deformation potentials, phonon energies) for the different scattering rates are also obtained from the literature. ${ }^{37,44,46}$ The graphene layer is assumed to be a perfect $2 \mathrm{D}$ honeycomb completely ideal, with no rippling, wrinkling or impurities. Acoustic and optical scattering processes, including TA, LA, TO and LO phonon branches, are incorporated. In order to account for degeneracy effects, the Pauli exclusion principle is considered. After selection of each scattering mechanism, a rejection technique based on the instantaneous wavevector distribution (updated each time step) is applied. ${ }^{29,30,47}$ More details about our Monte Carlo model can be found in previous works. ${ }^{38,48}$ The drift velocity values obtained under high-field conditions (ranging from $\sim 4.4 \cdot 10^{7} \mathrm{~cm} / \mathrm{s}$ to $\sim 3.5 \cdot 10^{7} \mathrm{~cm} / \mathrm{s}$ for fields between $20-50 \mathrm{kV} / \mathrm{cm}$ for the carrier concentration range considered) are in good agreement with those presented in the literature in simulations and experimental measurements. ${ }^{30,32,46,49}$ Extremely high mobilities are found at low fields $\left(1.2 \cdot 10^{6}\right.$ to $3.7 \cdot 10^{5} \mathrm{~cm}^{2} / \mathrm{Vs}$ for carrier densities between $5 \cdot 10^{11} \mathrm{~cm}^{-2}$ and $\left.2 \cdot 10^{12} \mathrm{~cm}^{-2}\right)$, in the same order of magnitude of those from experimental data and modelling results in suspended graphene. ${ }^{44,50-54}$ Some authors point towards a reduced low-field mobility in suspended single layer graphene due to flexural phonon modes and imperfections in fabricated samples; ${ }^{49,55}$ since there is still controversy regarding this issue, our model accounts for the most favourable scenario for a suspended graphene layer.

To determine the diffusion coefficient, two carrier populations -background and excess carriers, as described in subsection II B- coupled via a exchange mechanism are considered. In the simulation, the events undergone by each single particle are recorded, thus providing not only the average values of velocity, energy or wavevector and their distribution functions, but also the average number of scatterings, the scattering time, the instantaneous velocity values, correlation functions, mean free paths, etc.

\section{B. Diffusion coefficient calculation}

The zero-field value of the diffusion coefficient (the diffusivity $D$ ) can be related to the low-field mobility through the Einstein relationship: ${ }^{56-58}$

$$
\mu=\frac{q D}{n_{S}} \frac{d n_{S}}{d E_{F}}
$$

where $\mu$ is the mobility, $q$ the elementary electron charge, $n_{S}$ the carrier concentration and $E_{F}$ the Fermi level. In the case of graphene, the Fermi level can be obtained through: ${ }^{3}$

$$
E_{F}=\hbar v_{F} \sqrt{\pi n_{S}}
$$

From equations (1) and (2) is is straightforward to get:

$$
D=\frac{\mu E_{F}}{2 q}
$$

From the classical motion of electrons in a diffusive random walk scattering independently off the different impurities, D. Sarma et al. ${ }^{3}$ provided the following alternative expression to determine the diffusion coefficient in graphene:

$$
D=\frac{v_{F}^{2} \tau}{2}=\frac{v_{F} \lambda}{2}
$$

where $\tau$ is the scattering relaxation time and $\lambda$ is the mean free path.

Under non-equilibrium conditions alternative ways of determining the diffusion coefficient (which becomes no longer isotropic) must be considered. In our case, the diffusion coefficient has been computed by means of two separate techniques, both based on microscopic stochastic simulations. The first one is focused on the second central moment (related to the spreading of the electron concentration profile) and the second one on the study of velocity fluctuations (essentially due to thermal noise processes). ${ }^{39}$

Considering the phenomenological definition of diffusion according to the Fick laws, ${ }^{59}$ at relatively large 
times, the diffusion coefficient corresponds to the variance of the random distances travelled by individual carriers per unit time, ${ }^{39}$ and can be obtained from the second central moment of the carrier distribution function in the following way: ${ }^{39,59}$

$$
D_{\|(\perp)}(E)=\frac{\left\langle\left(r_{\|(\perp)}-\left\langle r_{\|(\perp)}\right\rangle\right)^{2}\right\rangle}{2 t}
$$

where $E$ is the applied electric field, $t$ is the time, and $r_{\|(\perp)}$ corresponds to the parallel (perpendicular) position of each particle with regard to the applied electric field direction. The brackets denote ensemble average.

Due to the importance of the Pauli exclusion principle in graphene for the carrier densities considered, the calculations are performed considering an excess carrier population, as described in the literature in the case of a $2 \mathrm{DEG}^{59}$ or in degenerate bulk semiconductors. ${ }^{42,60} \mathrm{In}$ the simulation, two sets of particles are taken into account: the background carriers (the standard carrier concentration), obeying the non-linear Boltzmann transport equation (BTE), and an excess carrier population, small enough to be considered a minor perturbation of the system ( $\sim 10 \%$ of the background carriers), that evolves according to a linearized BTE. ${ }^{60}$ In this way, the calculations are performed for the excess carrier population, which is the one adequate to determine the diffusivity in a Monte Carlo simulation when degeneracy plays an important role, as in the present case. The number of background particles is $10^{5}$, while the number of excess particles is $10 \%$ of the number of background particles. This allows obtaining accurate results for the study of velocity fluctuations while not severely compromising the CPU time and computer resources. The coupling between both sets of populations is considered through a rejection technique based on a carrier-carrier exchange scattering mechanism. ${ }^{42,59,60}$ To deal with the particularities of graphene, the corresponding density of states ${ }^{3}$ is considered to determine the initial distributions of both background and excess electrons.

The second calculation method to obtain the diffusion coefficient is related to the Fourier analysis of instantaneous velocity fluctuations. ${ }^{61}$ The correlation function of velocity fluctuations can be obtained as:

$$
C_{v_{\|(\perp)}}(t)=\left\langle\delta v_{\|(\perp)}\left(t^{\prime}\right) \delta v_{\|(\perp)}\left(t^{\prime}+t\right)\right\rangle
$$

where $C_{v_{\|(\perp)}}$ is the time-dependent correlation function of parallel (perpendicular) velocity fluctuations, $\delta v_{\|(\perp)}$ the instantaneous fluctuation of parallel (perpendicular) velocity of individual particles and the brackets indicate ensemble average. Applying the Wiener-Kintchine theorem, the frequency-dependent diffusion coefficient is given by:

$$
D_{\|(\perp)}(\omega)=\frac{1}{4} S_{v_{\|(\perp)}}(\omega)=\int_{0}^{\infty} C_{v_{\|(\perp)}}(t) e^{j \omega t} d t
$$

where $S_{v_{\|(\perp)}}(\omega)$ is the frequency-dependent power spectral density of the parallel (perpendicular) velocity fluctuations. In this case, the calculations are performed also over the excess carrier concentration, following the methodology previously discussed.

For comparison purposes and in order to provide a more detailed and clarifying discussion, in some cases the results obtained for the background population are also presented.

\section{RESULTS AND DISCUSSION}

The diffusion coefficient as a function of the applied electric field, both for parallel and perpendicular directions with regard to the applied field and for background and excess electrons is presented in Figure 1. Grey and black symbols represent the values obtained with the second central moment calculation (equation 5), while white symbols account for diffusion coefficients determined from the low-frequency power spectral density of velocity fluctuations (equation 7), presented just for a few electric field values for comparison purposes. The yellow diamond indicates the value obtained for the diffusion coefficient from equation (3) taking into account the mobility extracted from the velocity-field curve at extremely low electric fields. ${ }^{38}$ The agreement between the diffusion coefficient obtained by means of the two methodologies considered (second central moment method and the velocity fluctuations method) is very good in the whole field range and in all the cases considered, both for background and excess carriers and for the parallel and perpendicular directions, so from now on we will focus on the discussion of the results provided by the second central moment. It is important to notice the important variation in the diffusion coefficient values from low to high electric fields, at least three orders of magnitude in the electric field range considered. This is consistent with an increase of the electron mass with the average energy at high fields, derived from the unique properties of the linear dispersion relation in graphene. ${ }^{30,38}$

However, if one examines the diffusion coefficient values at low electric fields (see inset of Figure 1) significant differences appear between background and excess carrier populations. The calculation from background carriers produces noticeably high and anisotropic values for both the parallel and perpendicular directions, much larger than the value predicted by the Einstein relationship (yellow diamond). However, for excess carriers, at very low electric fields the parallel and perpendicular diffusion coefficients tend to become similar (thus making the diffusion coefficient isotropic) and get very close to the equilibrium value predicted by the Einstein relationship. Both trends are similar to the results obtained by other authors in the case of bulk degenerate semiconductors and 2DEG. ${ }^{42,59,60}$

To get a better understanding of the behaviour of diffusivity and carrier transport, the wavevector distribution function of background and excess carriers is presented in Figure (2). At extremely low electric fields (Figure 2(a), $E=0.01 \mathrm{kV} / \mathrm{cm}$ ), the background pop- 


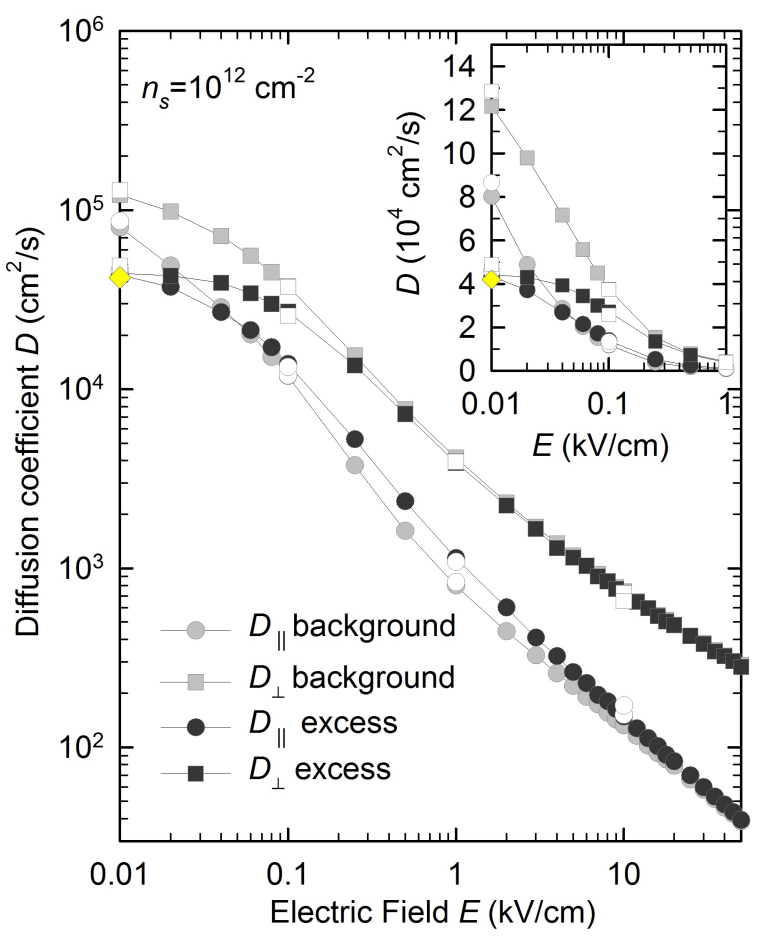

FIG. 1. Diffusion coefficient for parallel (circles) and perpendicular (squares) directions for background (grey) and excess (black) carriers obtained by means of the second central moment. White symbols show the results obtained from velocity fluctuations just for some selected electric field values in order to provide an insight of the agreement of both methods and avoid excessive symbol overlapping in the figure. The inset shows an ampliation of the low-field region. The carrier density is equal to $10^{12} \mathrm{~cm}^{-2}$.

ulation is practically restricted to the circle set by the Fermi wavevector, with just a very small deviation from the origin. In the case of excess electrons (Figure 2(d)), at this ultra-low $E$ they are distributed at the $k$ states near the surface defined by the Fermi wavevector, not being allowed to occupy the lowest $k$ values already fulfilled by background carriers (Figure 2(d)). This is to be expected since the excess carrier distribution corresponds to the incremental distribution function with regard to $n_{S}$. Close to equilibrium, for both populations quasi-elastic acoustic scattering (Figure 3(a)) is the dominant mechanism ( $~ 66 \%$ of the total number of mechanisms). The differences appear regarding inelastic scattering events: optical and acoustic intervalley emission processes are clearly more relevant for the excess carrier population, in good agreement with its larger average energy at that electric field $(126.7 \mathrm{meV}$ for excess carriers and $94.2 \mathrm{meV}$ for background carriers, see Figure $3(\mathrm{~d})$ ) and with the wavevector distribution function obtained. Consequently, in this regime of very low electric field the background population fails to predict the diffusion coefficient since most carriers travel without suffering scatterings in the Fermi region (they are forbidden by

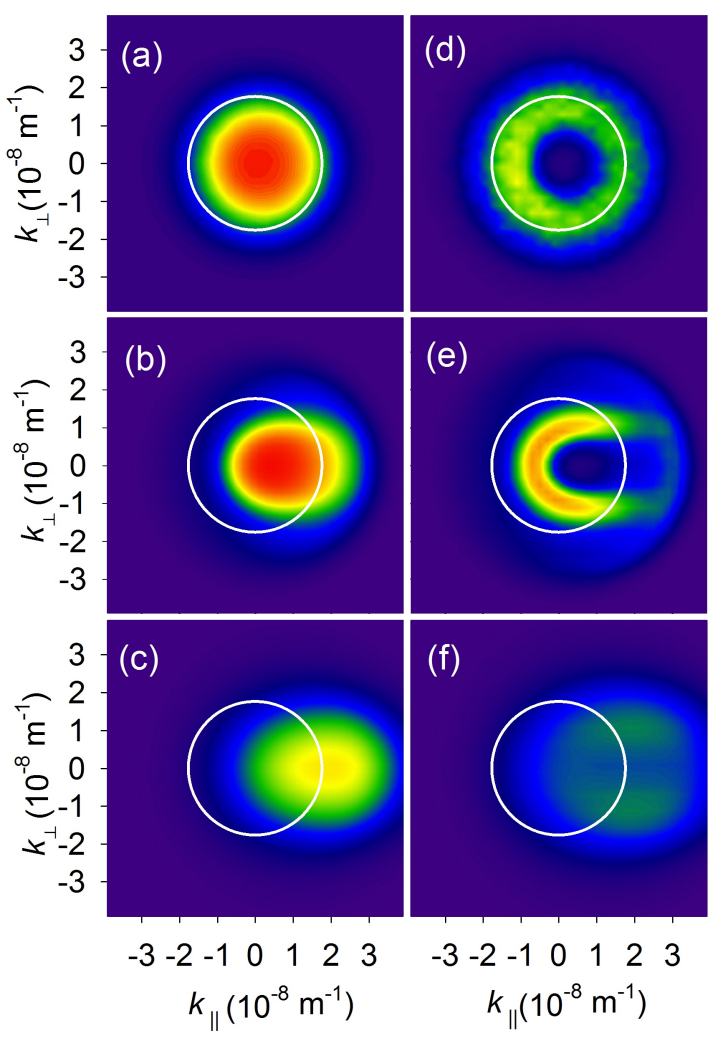

FIG. 2. Wavevector distribution function for background carriers at $E=0.01 \mathrm{kV} / \mathrm{cm}(\mathrm{a}), 0.1 \mathrm{kV} / \mathrm{cm}$ (b) and $1 \mathrm{kV} / \mathrm{cm} \mathrm{(c)}$ and excess carriers distribution at $E=0.01 \mathrm{kV} / \mathrm{cm}(\mathrm{d}), 0.1$ $\mathrm{kV} / \mathrm{cm}$ (e) and $1 \mathrm{kV} / \mathrm{cm}$ (f). The carrier density is equal to $10^{12} \mathrm{~cm}^{-2}$. The circles indicate the equilibrium Fermi surface as a reference.

the Pauli exclusion principle ${ }^{59}$ ) and therefore yield much larger scattering times (see Figure 4(a)). These scattering times for the background population are in the same order of magnitude that the carrier relaxation times experimentally observed in graphene $(\sim 20 \mathrm{ps}) .{ }^{53}$ In the case of excess electrons, there is a comparatively larger number of scattering events at low electric fields (174 $\%$ larger in the population of excess electrons as compared to background electrons at extremely low fields for $n_{S}=10^{12} \mathrm{~cm}^{-2}$ ) due to the reduced importance of Pauli exclusion principle at the edge of the Fermi surface. These two facts (transport at the edge of the Fermi surface and greater importance of inelastic phonon emission) combine to provide saturation and high isotropy of the diffusion coefficient values at extremely low applied fields, in good agreement with the Einstein relation.

As the electric field in increased to the $0.1 \mathrm{kV} / \mathrm{cm}$ range, the influence of intravalley acoustic phonons tends to reduce in both cases, being more significant the reduction in their percentage in the case of excess electrons. Conversely, optical phonon emission gets much influent, particularly for excess carriers with positive and large values of $k_{\|}$. While the distribution of background carriers is 


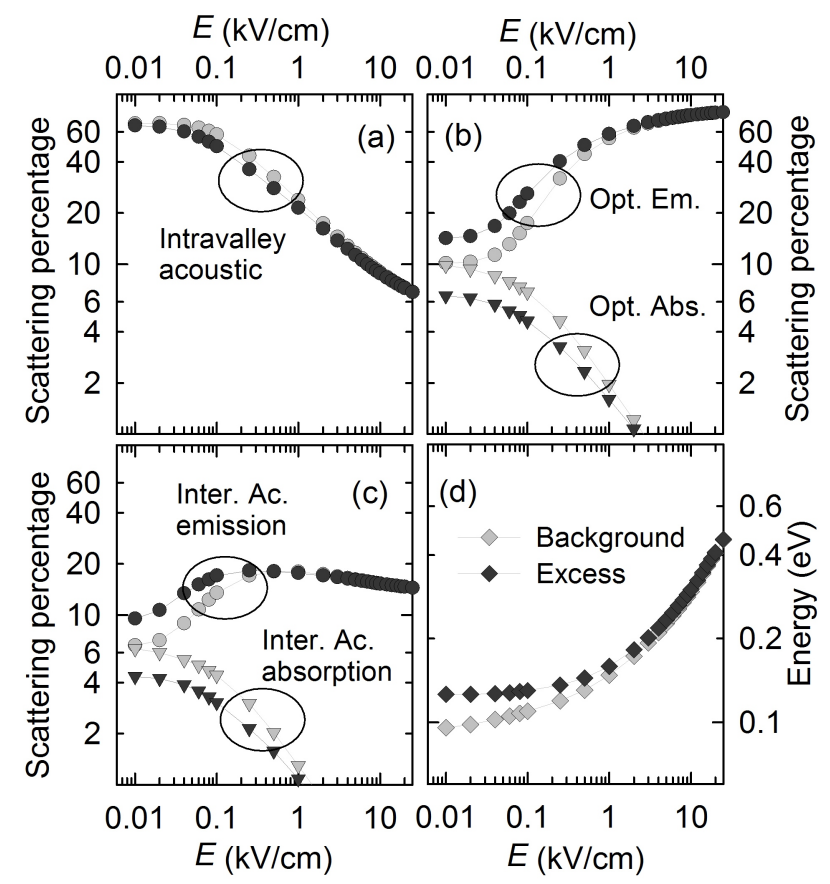

FIG. 3. Percentage of scattering events as a function of the applied field for background carriers (grey symbols) and excess carriers (black symbols) for $n_{S}=10^{12} \mathrm{~cm}^{-2}$ (a), (b) and (c). Average electron energy as a function of the applied field for background (grey diamonds) and excess (black diamonds) carriers (d).

spread towards the direction of the applied field (Figure 2 (b), $E=0.1 \mathrm{kV} / \mathrm{cm}$ ), the excess electrons wavevector distribution shows a $\subset$ shape (Figure 2(e)). Those excess electrons corresponding to the negative $k_{\|}$half distribution at equilibrium keep in a semicircle distribution, since the immediately higher $k_{\|}$states are still largely occupied by background electrons. The positive $k_{\|}$half at equilibrium is now largely displaced towards the right, thanks to the larger amount of free $k$ states available in that direction. The excess distribution function is therefore strongly anisotropic at that electric field value, and the diffusion coefficient becomes anisotropic too.

At electric fields over $0.2-0.4 \mathrm{kV} / \mathrm{cm}$ optical emission becomes the dominant scattering mechanism (emission by intervalley acoustic phonon plays a secondary role as compared to intravalley acoustic scattering at low fields and intrinsic optical emission at high fields), and the related scattering times (Figure 4(a)) drop to the tenths of ps range at very high fields, with corresponding mean free paths near $100 \mathrm{~nm}$, consistent with the data found in the literature for hot carriers. ${ }^{62}$ This provokes a larger dispersion of the wavevector cloud, so the degeneracy effects become less relevant and the differences in the wavevector distribution functions for both populations tend to vanish (Figures 2(c) and (f)). Consequently, the diffusion coefficients are quite similar for background and excess carriers at high fields, particularly for the perpendicular

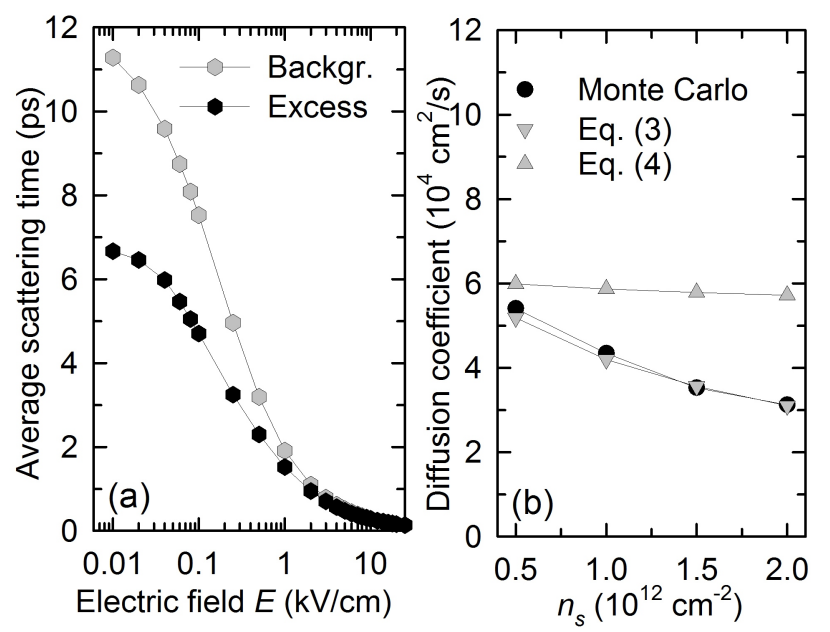

FIG. 4. Average time between scatterings for background (black circles) and excess carriers (grey circles) for $n_{S}=10^{12}$ $\mathrm{cm}^{-2}$ (a) and quasi-equilibrium diffusion coefficient as a function of the carrier density (circles: Monte Carlo results; triangles: results from equation (3); squares: results from equation (4) for background electrons and diamonds: for excess electrons).

direction and over $1 \mathrm{kV} / \mathrm{cm}$.

The dependence of the quasi-equilibrium diffusion coefficient as a function of the carrier density is shown in Figure 4(b). Together with the Monte Carlo results for the excess population at $E=0.01 \mathrm{kV} / \mathrm{cm}$, the results considering the Einstein relation (equation 3) and equation 4 are also presented. As it can be observed, the agreement between equation 3 and the Monte Carlo results is rather good, particularly at the highest values of $n_{S}$ presented. However, equation 4 clearly fails to predict not only the values of the diffusion coefficient at equilibrium, but also its dependence with the carrier concentration.

Let us now focus on the study of instantaneous velocity and its peculiarities in graphene. First, we present the average velocity distribution function of excess electrons (in the parallel and perpendicular directions) for three values of the electric field, $0.01,0.1$ and $1 \mathrm{kV} / \mathrm{cm}$ (Figure 5). As it can be observed, for all the applied fields and in both directions two local maximums appear for velocity values equal to $\pm v_{F}$. In the case of graphene, the maximum carrier velocity in any direction is limited by the carrier scalar velocity value, which is always equal to $v_{F}$ due to the fact that electrons behave as relativistic massless particles with constant velocity. ${ }^{1}$ Those peaks in the distribution function for parallel and perpendicular directions correspond therefore to electrons with their momentum strongly oriented in the $k_{\|}$or $k_{\perp}$ directions, respectively, with positive or negative sign. In the case of the perpendicular direction, the distribution is symmetric for positive and negative $v_{\perp}$ values, as it is to be expected from the wavevector distribution previously discussed. However, in the case of the parallel direction, as the electric field is raised, the distribution function tends 


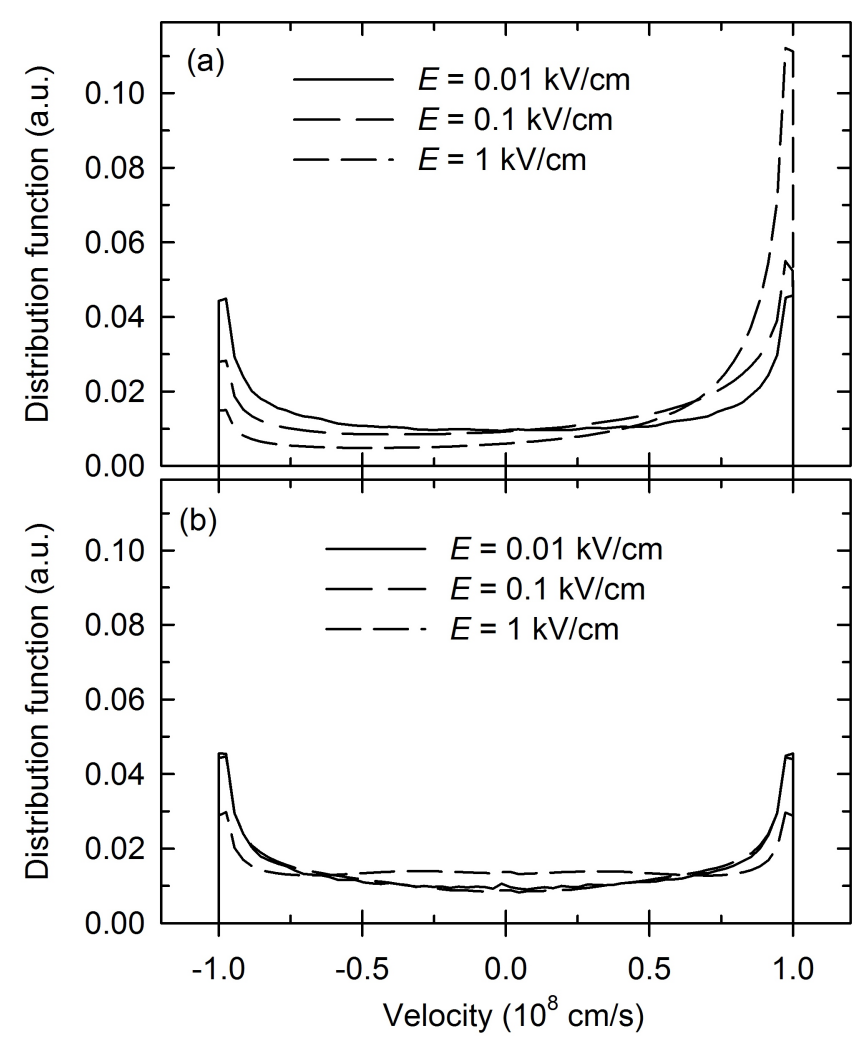

FIG. 5. Average velocity distribution function in the parallel (a) and perpendicular (b) directions for several values of the applied field (excess carriers).

to tilt towards $+v_{F}$, but not surpassing this value for the aforementioned reasons.

Consequently, the velocity fluctuations in graphene are limited an cannot reach arbitrarily high values. As an example, in Figure 6 depicts the instantaneous velocity fluctuations of a single particle as a function of time. Contrarily to the case of conventional semiconductors, in which parabolic-type bands imply a linear dependence of the velocity with time, the variation of the velocity component is not linear and shows positive and negative saturation values equal to $\pm v_{F}$. Therefore, even in the case of very large applied fields or under reduced scattering conditions the instantaneous velocity fluctuation would be always smaller than $2 v_{F}$.

The time-dependent autocorrelation function of parallel and perpendicular velocity fluctuations (equation 6) for the excess carrier population is shown in Figure 7 for several values of the applied electric field. For the lowest value of $E$, the results for the background carriers are also included for comparison purposes. At extremely low electric fields, the correlation function for excess carriers shows no relevant differences between parallel and perpendicular directions, which is consistent with the isotropic nature of the diffusivity in that regime. An exponential decay (in the form $C_{v}(t)=C_{v}(0) \exp (-t / \tau)$ ) is observed for these type of carriers. The background

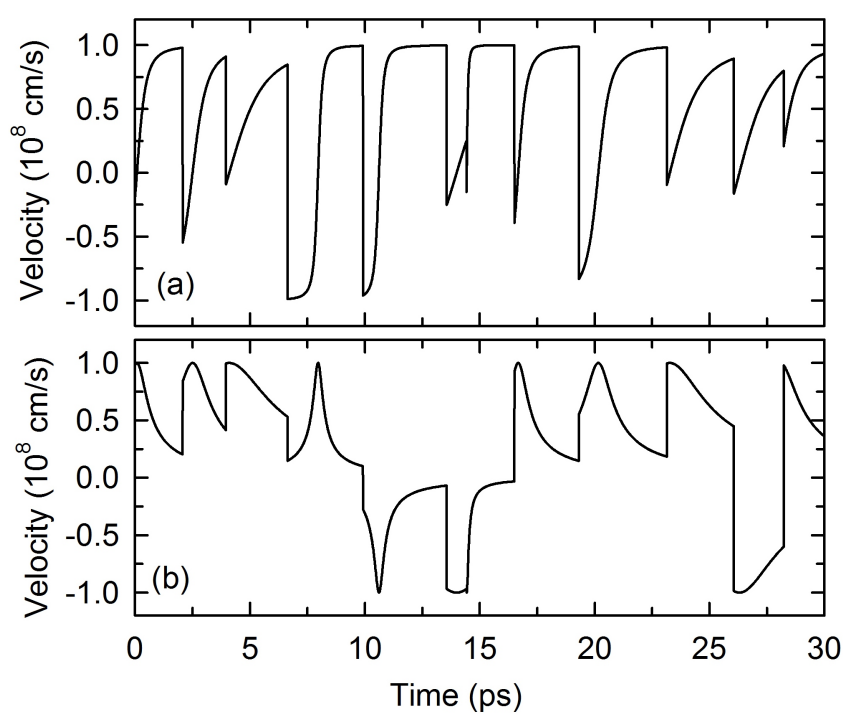

FIG. 6. Instantaneous velocity for a given particle in the parallel (a) and perpendicular (b) direction, for an applied field equal to $1 \mathrm{kV} / \mathrm{cm}$
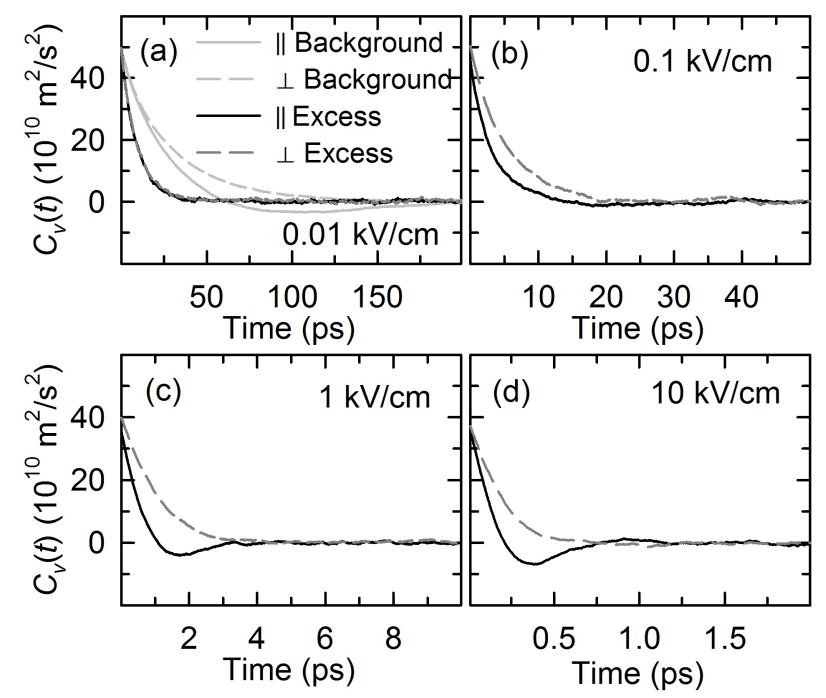

FIG. 7. Autocorrelation function of parallel and perpendicular velocity fluctuations for excess carriers, for $n_{S}=10^{12}$ $\mathrm{cm}^{-2}$ and several values of the applied electric field, 0.01 $\mathrm{kV} / \mathrm{cm}$ (a), $0.1 \mathrm{kV} / \mathrm{cm}$ (b), $1 \mathrm{kV} / \mathrm{cm}$ (c) and $10 \mathrm{kV} / \mathrm{cm}$ (d). Figure (a) includes also the results for the background carrier population for comparison.

carrier population, however, shows differences between both directions, with a slower decay in the perpendicular orientation, and the parallel one presenting negative values for $C_{v}(t)$ after several tens of ps. The reduced decay time of the correlation function for excess carriers is directly related to the minor influence of the Pauli exclusion principle and the increased emission of inelastic phonons previously discussed.

As the electric field is increased, some interesting ef- 
fects appear. First of all, the decay times become progressively shorter: in the case of the parallel direction, which shows an exponential decay with no negative parts in all cases, the characteristic times drop to the tenths of ps range for the highest electric field shown. The steady augmentation of the average carrier energy and the number of scatterings with $E$ acts as a correlation-breaker mechanism, reducing noticeably the correlation times. Differences also arise regarding the parallel and perpendicular orientations. While the perpendicular orientation keeps the previously mentioned exponential decay shape observed at the lowest electric field, the parallel correlation function shows a negative peak, particularly noticeable for $E \geq 1 \mathrm{kV} / \mathrm{cm}$. This is related to the strong anisotropy of the wavevector distribution evidenced in Figure 2. The distribution is symmetric regarding the $k_{\perp}$ axis but it is asymmetric with regard to the $k_{\|}$axis, since the centroid position is displaced in the parallel direction under the action of the applied field. To understand the consequences of this behaviour, it is important to remind that, as discussed above, in graphene the velocity of carriers is always equal to the Fermi velocity, and their $v_{\|}$and $v_{\perp}$ values are determined by the ratio between the wavevector components and the wavevector module. During free flights, the $k_{\perp}$ value for a given carrier remains constant due to the absence of a $E_{\perp}$ field, and consequently the $v_{\perp}$ velocity usually decreases but does not change its sign with regard to the initial flight time (see Figure 6(b)). Hence, the sign of the $v_{\perp}$ fluctuation for every single carrier remains the same and the correlation is always positive. The decay is faster at higher fields since in this case the decrease of $v_{\perp}$ during the free flight is quicker because of the rapid increase of the wavevector module, and consequently the fluctuation (the average $v_{\perp}$ value is zero) also decreases.

Concerning the parallel direction, the asymmetric distribution of the wavevector is a key fact to understand the appearance of a negative peak in $C_{v_{\|}}(t)$. Carriers with an initial $v_{\|}$value lower than the average (and consequently, with a negative initial velocity fluctuation) progressively increase their $v_{\|}$under the action of the applied field, and can easily turn their fluctuation into positive when they surpass the average $v_{\|}$value. Carriers with a parallel velocity higher than the average (with initial positive fluctuation) have, on the one hand, a limitation of the increase of the fluctuation value since they rapidly approach the $v_{F}$ limit and, on the other hand, have a large probability of being scattered back to lower energy values (and $v_{\|}$below the average) by isotropic inelastic scattering (intrinsic optical and intervalley acoustic emission). Consequently, in both cases (carriers over and below the average $v_{\|}$value) there is a very elevated probability of changing their fluctuation sign, therefore producing negative $C_{v_{\|}}(t)$ values after a relatively short time. This is another important difference with regard to conventional semiconductors with negative differential conductance, in which the negative peak of the correlation function is explained in terms of partition noise, due to the transfer to

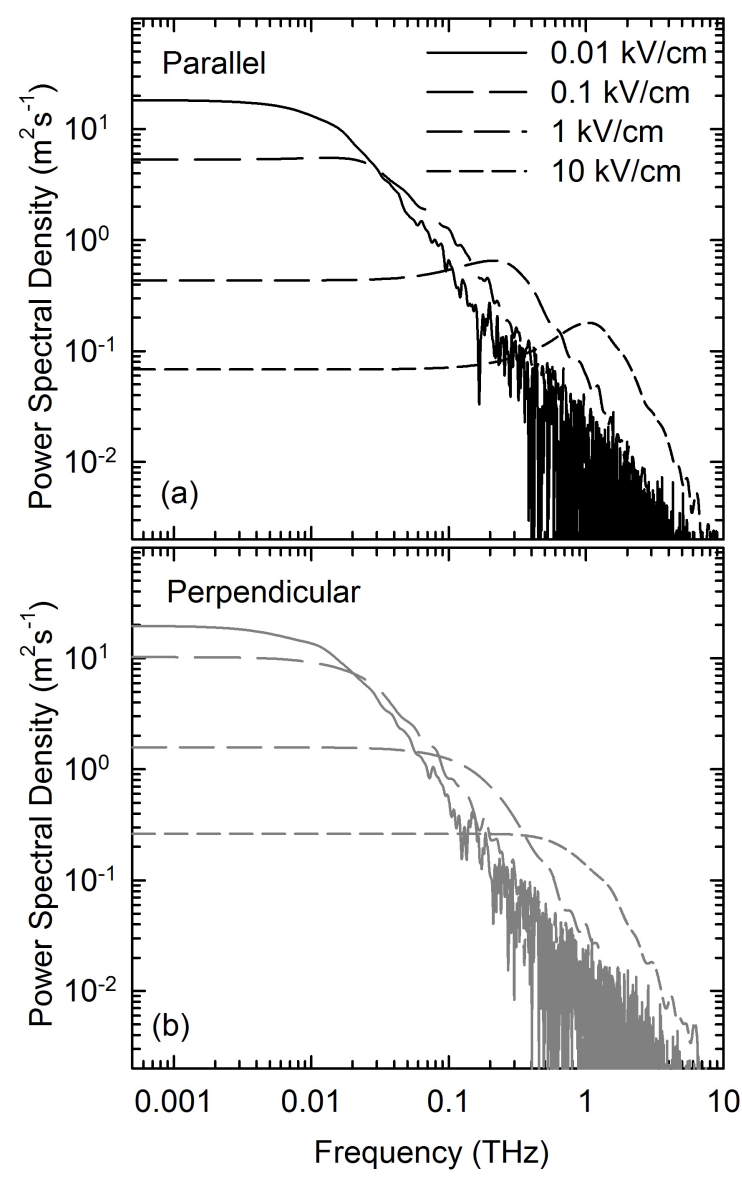

FIG. 8. Parallel (a) and perpendicular (b) power spectral density of velocity fluctuations for $n_{S}=10^{12} \mathrm{~cm}^{-2}$ and several values of the applied electric field.

upper valleys in the conduction band. ${ }^{39}$

The power spectral density of velocity fluctuations $\left(S_{v}(f)\right)$ of excess carriers as a function of the frequency, up to $10 \mathrm{THz}$, is shown in Figure 8 both for parallel and perpendicular directions and several values of the applied electric field. This quantity provides information for the power dissipated by velocity fluctuations at a given frequency. In the case of parallel velocity fluctuations (Figure 8(a)) a white noise behaviour is observed in all cases in the $\mathrm{GHz}$ range. As the electric field is increased, the low field value is reduced, the white noise region extends to higher frequencies (consistent with the progressively shorter correlation times) and a peak appears in the $\mathrm{THz}$ range for $S_{v_{\|}}(f)$ (approximately $1.04 \mathrm{THz}$ at $10 \mathrm{kV} / \mathrm{cm}$ ) related to the negative peak of the correlation function. At high frequencies there is a monotonic decrease of the $S_{v}(f)$ values in all cases.

For perpendicular velocity fluctuations (Figure 8(b)) the low frequency values of $S_{v_{\perp}}$ are larger than those of the parallel direction, particularly at high fields. This is in good agreement with the previously discussed larger diffusivity in that orientation, and indicates a greater power dissipation by velocity fluctuations in the perpen- 
dicular direction. In this case, no local maximum is observed in the $\mathrm{THz}$ range, consequence of the monotonic correlation decay produced by the microscopic fluctuations in this direction. In all cases, there is a white noise plateau followed by a monotonic decay, the corner frequency being displaced towards larger frequencies as $E$ is increased. For $E=10 \mathrm{kV} / \mathrm{cm}$ the plateau extends practically up to $1 \mathrm{THz}$, corresponding to extremely fast (tenths of ps) correlation times.

It is important to remark that the results presented correspond to an ideal, defect-free, suspended graphene layer electrically excited. In the case of optically pumped excitations, interband interactions shall play a fundamental role in the carrier dynamics at the $\mathrm{THz}$ range. ${ }^{63,64}$ Moreover, shot noise due to carriers traversing potential barriers could also have influence at least at the $\mathrm{GHz}$ range, as recently shown by other authors. ${ }^{18}$

\section{CONCLUSIONS}

The diffusion coefficient in monolayer graphene and its evolution with the applied electric field has been investigated by means of an ensemble Monte Carlo simulator. Two methods have been considered: the second central moment calculation, based on the spreading of the carrier population, and the Fourier analysis of the instantaneous velocity fluctuations. In the simulation the effect of degeneracy on the diffusion at a microscopic level is carefully taken into account by means of the consideration on an excess carrier population (coupled to background carriers through a carrier-carrier exchange mechanism) which obeys a linearized Boltzmann transport equation.

The results show that in order to obtain physically sound values of the diffusion coefficient (in agreement with the Einstein relation in equilibrium and the isotropic nature of the diffusion coefficient at extremely low electric fields) the calculations shall be performed over the excess carriers travelling at the edge of the Fermi surface, which are those relevant for the consideration of diffusion phenomena. It is also shown that for the case of graphene the expression of the diffusion coefficient in terms of the scattering times or mean free path for standard carriers fails to predict not only the diffusion coefficient value, but also its dependence on the carrier density. A very important decay of the diffusion coefficient with the applied electric field in suspended monolayer graphene has been also observed, falling three orders of magnitude from the equilibrium value to high field conditions.

The correlation function of velocity fluctuations has been also investigated. Its behaviour and dependence on the electric field has been explained in terms of the microscopic nature of fluctuations, with special attention to the different conditions for parallel and perpendicular directions with regard to the applied field and the microscopic wavevector distribution of carriers. While the correlation function for perpendicular fluctuations presents a monotonic decay and larger correlation times, the fluc- tuations in the parallel direction show a negative peak in its correlation function consequence of the distribution of the wavevector population under the applied electric field and the fluctuation limitations imposed by the Fermi velocity of carriers. The power spectral density of velocity fluctuations evidences a white noise behaviour in the $\mathrm{GHz}$ range, and a monotonic decay in the $\mathrm{THz}$ range. Parallel velocity fluctuations have a reduced power dissipation, but show a maximum in the values of the spectral density which takes place around $1 \mathrm{THz}$ for high electric fields, which is related to the observed correlation times, in the tenths of picoseconds range.

\section{ACKNOWLEDGMENTS}

This work has been supported by research project SA188A11 from the Consejería de Educación de la Junta de Castilla y León.

${ }^{1}$ K. S. Novoselov, A. K. Geim, S. V. Morozov, D. Jiang, Y. Zhang, S. V. Dubonos, I. V. Grigorieva, and A. A. Firsov, Science 306, 666 (2004).

${ }^{2}$ K. S. Novoselov, V. I. Fal'ko, L. Colombo, P. R. Gellert, M. G. Schwab, and K. Kim, Nature 490, 192 (2012).

${ }^{3}$ S. Das Sarma, S. Adam, E. H. Hwang, and E. Rossi, Rev. Mod. Phys. 83, 407 (2011).

${ }^{4}$ F. Schwierz, Nat. Nano 5, 487 (2010).

${ }^{5}$ L. Liao, Y.-C. Lin, M. Bao, R. Cheng, J. Bai, Y. Liu, Y. Qu, K. L. Wang, Y. Huang, and X. Duan, Nature 467, 305 (2010).

${ }^{6}$ L. Liao, J. Bai, R. Cheng, Y.-C. Lin, S. Jiang, Y. Qu, Y. Huang, and X. Duan, Nano Letters 10, 3952 (2010).

${ }^{7}$ J. Zheng, L. Wang, R. Quhe, Q. Liu, H. Li, D. Yu, W.-N. Mei, J. Shi, Z. Gao, and J. Lu, Sci. Rep. 3:1314 (2013), 10.1038/srep01314.

${ }^{8}$ S. Das and J. Appenzeller, in Radio Frequency Integrated Circuits Symposium (RFIC), 2011 IEEE (2011) pp. 1-4.

${ }^{9}$ W. Knap, S. Rumyantsev, M. S. Vitiello, D. Coquillat, S. Blin, N. Dyakonova, M. Shur, F. Teppe, A. Tredicucci, and T. Nagatsuma, Nanotechnology 24, 214002 (2013).

${ }^{10}$ T. Otsuji, S. A. B. Tombet, A. Satou, H. Fukidome, M. Suemitsu, E. Sano, V. Popov, M. Ryzhii, and V. Ryzhii, Journal of Physics D: Applied Physics 45, 303001 (2012).

${ }^{11}$ T. Otsuji, T. Watanabe, S. Boubanga Tombet, A. Satou, W. Knap, V. Popov, M. Ryzhii, and V. Ryzhii, Terahertz Science and Technology, IEEE Transactions on 3, 63 (2013).

${ }^{12}$ A. A. Dubinov, V. Y. Aleshkin, V. Mitin, T. Otsuji, and V. Ryzhii, Journal of Physics: Condensed Matter 23, 145302 (2011).

${ }^{13}$ G. Iannaccone, A. Betti, and G. Fiori, in Noise and Fluctuations (ICNF), 2011 21st International Conference on (2011) pp. 360363.

${ }^{14}$ S. Rumyantsev, G. Liu, W. Stillman, M. Shur, and A. A. Balandin, Journal of Physics: Condensed Matter 22, 395302 (2010).

${ }^{15}$ J. S. Moon, D. Curtis, D. Zehnder, S. Kim, D. K. Gaskill, G. Jernigan, R. L. Myers-Ward, J. Eddy, C.R., P. M. Campbell, K.-M. Lee, and P. Asbeck, Electron Device Letters, IEEE 32, 270 (2011).

${ }^{16}$ A. N. Pal, S. Ghatak, V. Kochat, E. S. Sneha, A. Sampathkumar, S. Raghavan, and A. Ghosh, ACS Nano 5, 2075 (2011).

${ }^{17}$ Y. Zhang, E. E. Mendez, and X. Du, ACS Nano 5, 8124 (2011).

${ }^{18}$ L. Ardaravicius, J. Liberis, E. Sermuksnis, A. Matulionis, J. Hwang, J. Y. Kwak, D. Campbell, H. A. Alsalman, L. F. Eastman, and M. G. Spencer, Physica Status Solidi (RRL) Rapid Research Letters 7, 348 (2013).

${ }^{19}$ M. Belonenko, N. Lebedev, and S. Sudorgin, Technical Physics 57, 1025 (2012). 
${ }^{20}$ B. A. Ruzicka, S. Wang, L. K. Werake, B. Weintrub, K. P. Loh, and H. Zhao, Phys. Rev. B 82, 195414 (2010).

${ }^{21}$ B. A. Ruzicka, S. Wang, J. Liu, K.-P. Loh, J. Z. Wu, and H. Zhao, Opt. Mater. Express 2, 708 (2012).

${ }^{22}$ K. Ziegler, Phys. Rev. B 79, 195424 (2009).

${ }^{23}$ T. Ando, NPG Asia Mater 1, 17 (2009).

${ }^{24}$ M. Ancona, IEEE Trans. Electron Dev. 57, 681 (2010).

${ }^{25}$ T. Stauber, N. M. R. Peres, and F. Guinea, Phys. Rev. B 76, 205423 (2007).

${ }^{26}$ S. Adam, P. W. Brouwer, and S. Das Sarma, Phys. Rev. B 79, 201404 (2009).

${ }^{27}$ D. Ferry, Journal of Computational Electronics 12, 76 (2013).

${ }^{28}$ A. Akturk and N. Goldsman, Journal of Applied Physics 103, 053702 (2008).

${ }^{29}$ J. Chauhan and J. Guo, Applied Physics Letters 95, 023120 (2009).

${ }^{30}$ R. S. Shishir and D. K. Ferry, Journal of Physics: Condensed Matter 21, 344201 (2009).

${ }^{31}$ M. Bresciani, P. Palestri, D. Esseni, and L. Selmi, Solid-State Electronics 54, 1015 (2010).

${ }^{32}$ X. Li, E. A. Barry, J. M. Zavada, M. B. Nardelli, and K. W. Kim, Applied Physics Letters 97, 082101 (2010).

${ }^{33}$ T. Fang, A. Konar, H. Xing, and D. Jena, Phys. Rev. B 84, 125450 (2011).

${ }^{34}$ B. Ghosh and S. Misra, Journal of Applied Physics 110, 043711 (2011).

${ }^{35}$ N. Harada, Y. Awano, S. Sato, and N. Yokoyama, Journal of Applied Physics 109, 104509 (2011).

${ }^{36}$ E. Sano, Applied Physics Express 4, 085101 (2011).

${ }^{37}$ J. K. David, L. Register, and S. Banerjee, Electron Devices, IEEE Transactions on 59, 976 (2012).

${ }^{38} \mathrm{R}$. Rengel, C. Couso, and M. Martin, in Electron Devices (CDE), 2013 Spanish Conference on (2013) pp. 175-178.

${ }^{39}$ R. Fauquembergue, J. Zimmermann, A. Kaszynski, and E. Constant, Journal of Applied Physics 51, 1065 (1980).

${ }^{40}$ R. Brunetti, C. Jacoboni, F. Nava, L. Reggiani, G. Bosman, and R. J. J. Zijlstra, Journal of Applied Physics 52, 6713 (1981).

${ }^{41}$ T. G. Sánchez, J. E. V. Pérez, P. M. G. Conde, and D. P. Collantes, Journal of Applied Physics 72, 2322 (1992).

${ }^{42}$ P. Borowik and J. L. Thobel, Semiconductor Science and Technology 14, 450 (1999).

${ }^{43}$ H.-S. P. Wong and A. D., Carbon nanotube and graphene device physics, 1st ed. (Cambridge University Press, 2011).

${ }^{44}$ K. M. Borysenko, J. T. Mullen, E. A. Barry, S. Paul, Y. G. Semenov, J. M. Zavada, M. B. Nardelli, and K. W. Kim, Phys. Rev. B 81, 121412 (2010).

${ }^{45}$ E. H. Hwang and S. Das Sarma, Phys. Rev. B 77, 115449 (2008).

${ }^{46}$ X. Li, E. A. Barry, J. M. Zavada, M. B. Nardelli, and K. W.
Kim, Appl. Phys. Lett. 97, 232105 (2010).

${ }^{47}$ P. Lugli and D. Ferry, Electron Devices, IEEE Transactions on 32, 2431 (1985).

${ }^{48}$ M. Martin, C. Couso, and R. Rengel, in 22nd International Conference on Noise and Fluctuations (ICNF 2013) (2013) p. In press.

${ }^{49}$ V. E. Dorgan, A. Behnam, H. J. Conley, K. I. Bolotin, and E. Pop, Nano Letters (2013), 10.1021/nl400197w.

${ }^{50}$ S. V. Morozov, K. S. Novoselov, M. I. Katsnelson, F. Schedin, D. C. Elias, J. A. Jaszczak, and A. K. Geim, Phys. Rev. Lett. 100, 016602 (2008).

${ }^{51}$ K. I. Bolotin, K. J. Sikes, J. Hone, H. L. Stormer, and P. Kim, Phys. Rev. Lett. 101, 096802 (2008).

${ }^{52}$ K. Bolotin, K. Sikes, Z. Jiang, M. Klima, G. Fudenberg, J. Hone, P. Kim, and H. Stormer, Solid State Communications 146, 351 (2008).

${ }^{53}$ P. Neugebauer, M. Orlita, C. Faugeras, A.-L. Barra, and M. Potemski, Phys. Rev. Lett. 103, 136403 (2009).

${ }^{54}$ D. C. Elias, R. V. Gorbachev, A. S. Mayorov, S. V. Morozov, A. A. Zhukov, P. Blake, L. A. Ponomarenko, I. V. Grigorieva, K. S. Novoselov, F. Guinea, and A. K. Geim, Nature Phys. 7, 701 (2011).

${ }^{55}$ E. V. Castro, H. Ochoa, M. I. Katsnelson, R. V. Gorbachev, D. C. Elias, K. S. Novoselov, A. K. Geim, and F. Guinea, Phys. Rev. Lett. 105, 266601 (2010).

${ }^{56}$ N. W. Ashcroft and N. D. Mermin, Solid State Physics (Saunders College, 1976).

${ }^{57}$ B. R. Nag, Electron Transport in Compound Semiconductors (Springer London, 1980).

${ }^{58}$ G. I. Zebrev, "Graphene field effect transistors: Diffusion-drift theory," in Physics and Applications of Graphene - Theory, edited by S. Mikhailov (InTech, 2011).

${ }^{59}$ J. L. Thobel, A. Sleiman, and R. Fauquembergue, J. Appl. Phys. 82, 1220 (1997).

${ }^{60}$ P. Borowik and J. L. Thobel, Journal of Applied Physics 87, 329 (2000).

${ }^{61}$ C. Jacoboni and P. Lugli, The Monte-Carlo Method for Semiconductor Device Simulation (Springer, 1989).

${ }^{62}$ W.-K. Tse, E. H. Hwang, and S. D. Sarma, Applied Physics Letters 93, 023128 (2008).

${ }^{63}$ T. Winzer and E. Malic, Journal of Physics: Condensed Matter 25, 054201 (2013).

${ }^{64}$ S. Winnerl, F. Gttfert, M. Mittendorff, H. Schneider, M. Helm, T. Winzer, E. Malic, A. Knorr, M. Orlita, M. Potemski, M. Sprinkle, C. Berger, and W. A. de Heer, Journal of Physics: Condensed Matter 25, 054202 (2013). 